\title{
A numerical study of a plane wall jet with heat transfer
}

\author{
Iftekhar Z. Naqavi*, James C. Tyacke, Paul G. Tucker \\ Department of Engineering, University of Cambridge, Cambridge, UK
}

\section{A R T I C L E I N F O}

\section{Article history:}

Available online $\mathrm{xxx}$

\section{Keywords:}

Wall jet

Heat transfer

Direct numerical simulation (DNS)

Prandtl number

Turbulent Prandtl number

\begin{abstract}
A B S T R A C T
A direct numerical simulation (DNS) of a wall jet is performed at $R e=7500$. To the authors' knowledge, this is the highest Reynolds number DNS study of a wall jet. The heat transfer process is studied with an iso-thermal boundary condition at the wall. The molecular Prandtl number is $\operatorname{Pr}=0.71$. Mean flow and heat transfer parameters are contrasted with available measurements and Nusselt number coefficient correlations. The scaling parameters for heat transfer variables are investigated. The mean temperature $\langle T\rangle$, temperature root mean square $T_{r m s}$, streamwise $\left\langle u^{\prime} T^{\prime}\right\rangle$ and wall normal $\left\langle v^{\prime} T^{\prime}\right\rangle$ heat flux profiles show collapse in the streamwise direction, with the inner scaling, the outer scaling and the thermal scaling parameters. The complete budgets for temperature variance $\left\langle T^{\prime} T^{\prime}\right\rangle$ and turbulent heat fluxes are also presented.
\end{abstract}

(c) 2016 Published by Elsevier Inc.

\section{Introduction}

A high momentum fluid issuing from a narrow slot along a flat plate forms a wall jet. The near wall region, called the inner layer, acts like a turbulent boundary layer flow. The region away from the wall, called the outer layer, acts like a free shear flow. Due to its practical applications in film cooling for gas turbine blades and boundary layer control on high lift airfoils it has been studied extensively, Launder and Rodi provide a review of the state of the art until 1983 in Launder and Rodi (1983). Determination of self-similar behaviour in wall jets is important for turbulence modelling. The required eddy viscosity depends on the different flow regions and uncertainties in turbulent statistics have been found to be high (Launder and Rodi, 1983). It has been shown by George et al. (2000) that with appropriate scaling, velocity and Reynolds stress profiles collapse at infinitely large Reynolds number. There are two different scalings for the two wall jet regions, namely, the inner and outer scaling. These are presented in Fig. 1. For the inner layer, friction velocity $u_{\tau}$ and $v / u_{\tau}$ are the velocity and length scales. The outer scaling parameter for the velocity is the local maximum mean streamwise velocity $U_{\max }$ and for the wall normal distance it is $y_{1 / 2}$, which is the wall normal distance of a point where the mean streamwise velocity $\langle u\rangle$ is half of the $U_{\max }$. In the outer layer, Reynolds shear stress scales with friction velocity $u_{\tau}^{2}$, whereas normal stresses and mean velocities scale with maximum velocity $U_{\max }$.

\footnotetext{
* Corresponding author.

E-mail address: izn20@eng.cam.ac.uk (I.Z. Naqavi).
}

Several studies with measurements and simulations are available, investigating the flow physics of wall jets (Launder and Rodi, 1983). The heat transfer from an isothermal wall, which is an important aspect of the wall jet applications, has received little attention. There is significant variation in suggested constants for log-law $\left(\frac{1}{\kappa} \ln y^{+}+A\right)$ type flow behaviour in planar wall jets (for example $0.41<\kappa<0.6$ and $5<A<6.8$ (Banyassady and Piomelli, 2015)) and this poses a problem for flow and thermal predictions and measurements. Ahlman et al. (2007) performed a DNS of a wall jet with scalar transport, at a relatively lower Reynolds number of 2000 to study inner and outer scalings showing self similarity behaviour at several downstream locations. Banyassady and Piomelli (2015) use LES and joint probability density functions to assess the level of influence of the outer layer on the inner. They conclude an independent scaling at infinite Reynolds number and a larger scaling overlap region as local Reynolds number decreases as suggested by George et al. (2000). For a $R e=9600$ wall jet, Dejoan and Leschziner (2005) compute turbulence budgets and realizability maps highlighting turbulent stresses, length and time scales to differ substantially from channel flows. These are important turbulence modelling aspects. They employ LES, finding minor subgrid model effects on an 8 million cell mesh. Dacos et al. (1984) measured temperature, heat fluxes and the triple-velocity-temperature product for a plane wall jet with isothermal boundary conditions. They note that over $90 \%$ of the temperature change from the wall is effected in the inner layer and compare temperature profile scalings using wall coordinates. For a planar wall jet, AbdulNour et al. (2000) measure the convective heat transfer coefficient. The authors focus on the developing flow region at smaller axial distances $(0<x / h<13)$ for automotive defroster applications. Insensitivity 


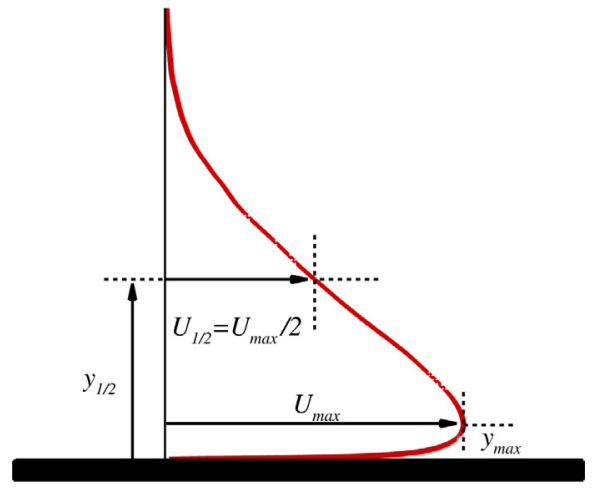

Fig. 1. Parameters for inner and outer scaling.

to the thermal boundary condition was found at locations $(5 \leq$ $x / h$ ), where the outer layer has diffused into the inner jet. A minimum in heat transfer coefficient is also found at $x / h \approx 5$. The correlation between the turbulence and heat transfer processes is also poorly understood. Pouransari et al. (2015) study the effect of passive and reactive scalar fields on the anisotropy of a wall jet using DNS. Anisotropy is accentuated near the wall but persists throughout the wall jet. Strong intermittency and anisotropy persistence at small scales is hence a challenge to predict.

In the current work a direct numerical simulation of a plane wall jet is performed to investigate the heat transfer process from an isothermal wall. The simulations are conducted at a significant Reynolds number, $R e=7500$ for which Rostamy et al. (2011) have performed flow measurements. The emphasis in this paper is on the scaling properties of the flow and heat transfer variables. In addition to the inner and outer scaling for heat transfer variables, the so called thermal scale is also considered. In thermal scaling, the thermal half width $y_{\theta / 2}$ is defined as the wall normal distance of a point where the temperature is half of the maximum local temperature. The results for velocity field, turbulent heat flux and their budgets will also be discussed and contrasted with other flows and literature.

\section{Simulation details}

The wall jet is simulated with the conservation of mass and momentum equations for unsteady three dimensional incompressible flow:

$$
\begin{aligned}
& \frac{\partial u_{j}}{\partial x_{j}}=0 \\
& \frac{\partial u_{i}}{\partial t}+\frac{\partial u_{j} u_{i}}{\partial x_{j}}=-\frac{\partial p}{\partial x_{i}}+\frac{1}{R e} \frac{\partial^{2} u_{i}}{\partial x_{j} \partial x_{j}}
\end{aligned}
$$

where $\left\{x_{1}, x_{2}, x_{3}\right\}=\{x, y, z\}$ are the coordinates in the streamwise, wall-normal and spanwise directions, respectively. $\left\{u_{1}, u_{2}, u_{3}\right\}=$ $\{u, v, w\}$ are the corresponding instantaneous velocities. $p$ is the instantaneous pressure. $R e=U_{j} h / v$ is the Reynolds number based on the jet velocity $U_{j}$, the jet slot height $h$ and the molecular viscosity $v$.

Heat transfer is simulated with a scalar transport equation:

$\frac{\partial T}{\partial t}+\frac{\partial T u_{i}}{\partial x_{i}}=\frac{1}{R e P r} \frac{\partial^{2} T}{\partial x_{i} \partial x_{i}}$,

where $T$ is the non-dimensional instantaneous temperature and $\mathrm{Pr}$ is the molecular Prandtl number. The non-dimensional temperature is defined as $T=\frac{\theta-\theta_{\infty}}{\theta_{w}-\theta_{\infty}}$, whereas $\theta$ is the physical temperature, $\theta_{w}$ is the wall temperature and $\theta_{\infty}$ is the temperature of the incoming fluid at the inlet plane. These governing equations are discretised with a second-order, collocated, finite volume solver. The solver is based on fractional step scheme, which uses semi-implicit time advancement. The scalar convection term is discretized with the QUICK (Quadratic Upstream Interpolation for Convective Kinetics) scheme (Leonard, 1979). Further details of numerical methods and examples of application of this code can be found in previous publications (Radhakrishnan et al., 2006; Naqavi et al., 2014).

The computational domain has the dimensions of $L_{x} / h=$ 43.0, $L_{y} / h=40.0$ and $L_{z} / h=9.0$ in the streamwise, wall-normal and spanwise directions, respectively. At the inflow plane a velocity profile is specified for the wall jet up to $y / h=1.0$ and the rest of the plane has a uniform co-flow of $0.06 U_{j}$. This uniform co-flow provides the fluid for the jet entrainment. The lower wall obeys the no slip and impermeability conditions. The upper boundary has a free slip boundary condition and a periodic condition is applied in the spanwise direction. At the outflow plane a convective boundary condition is applied as $\frac{\partial u_{i}}{\partial t}+U_{\text {con } v} \frac{\partial u_{i}}{\partial x}$. The convective velocity $U_{\text {conv }}$ is the mean streamwise velocity at the outflow plane. It is calculated as a running average. The initial transients are eliminated with weighted averaging in time given as;

$U_{\text {conv }}^{n+1}=\frac{\Delta t}{W_{t}}\left\langle u^{n}\right\rangle_{z}+\left(1-\frac{\Delta t}{W_{t}}\right) U_{\text {conv }}^{n}$,

$\Delta t$ is the time step size, $n$ is the number of time step, \langle\rangle$_{z}$ represents the spanwise averaging and $W_{t}$ is the averaging weight. When the simulation is started from a uniform flow, $W_{t}=10$ for initial $t^{*}=t U_{j} / h=200$ time units. Once the flow is developed, $W_{t}=100$ is used for the next $t^{*}=500$ time units. Finally, a simple running time average is used to calculate $U_{\text {conv }}^{n+1}$;

$U_{\text {conv }}^{n+1}=U_{\text {conv }}^{n}+\left(U_{\text {conv }}^{n}-\left\langle u^{n}\right\rangle_{z}\right) / n$.

At the jet inlet plane, Rostamy et al. (2011) did not provide any mean or turbulent velocities. Hence, based on the inlet configuration (Fig. 2(a)) given by Rostamy et al. (2011), a precursor RANS calculation is used to calculate the mean inlet velocity profile. To add small, time dependent perturbations at the inlet, a separate channel flow simulation is used. The channel dimensions are $2 \pi h \times h \times \pi h$, in the streamwise, wall-normal and spanwise directions, respectively. The periodic condition is defined in the streamwise and spanwise directions and no-slip condition at the top and bottom wall of the channel. The channel flow Reynolds number is $R e_{\text {channel }}=\frac{U_{\text {bulk }} h}{v}=7500$. The mean channel flow velocity is removed from the instantaneous channel flow field and the remaining fluctuations are scaled to give a turbulence intensity of less than $0.1 \%$. These fluctuations are superimposed on the RANS velocity profile. These help to initiate shear layer transition in the wall jet. Fig. 2(b) shows the resulting profiles of the mean velocity and Reynolds stress in the streamwise direction at the jet inlet.

The heat transfer Eq. (3) is solved for temperature $T$, with a periodic boundary condition in the spanwise direction. A uniform temperature $T_{\infty}=0.0$ is defined at the inlet plane. A convective boundary condition, as described previously for the flow equations, is used at the outflow plane. The lower wall has the isothermal condition of $T_{w}=1.0$ and the upper wall is adiabatic. The Reynolds number is $R e=7500$ and the Prandtl number is $\mathrm{Pr}=0.71$ for the current simulations. The domain is discretized with $1652 \times$ $344 \times 302$ grid points in the streamwise, wall-normal and spanwise directions, respectively, giving a total of 172 million cells. Fig. 3(a) shows the grid spacing in wall units, based on local friction velocity, along the streamwise direction, with a maximum of $\Delta x^{+}<10.5, \Delta y^{+}<0.7$ and $\Delta z^{+}<12.0$. The $\Delta y$ grid varies in both streamwise and wall-normal direction to follow the spreading of the jet. The contours of $\Delta y^{+}$in Fig. 3(b) show that the maximum $\Delta y^{+}<10.0$ in the active flow region. There are six points below $y^{+}=5$ and twelve points below $y^{+}=11$. The simulation is performed for $t^{*}=1300$ time units to remove the initial transients. The statistics are collected for next $t^{*}=1200$ time units. 
a

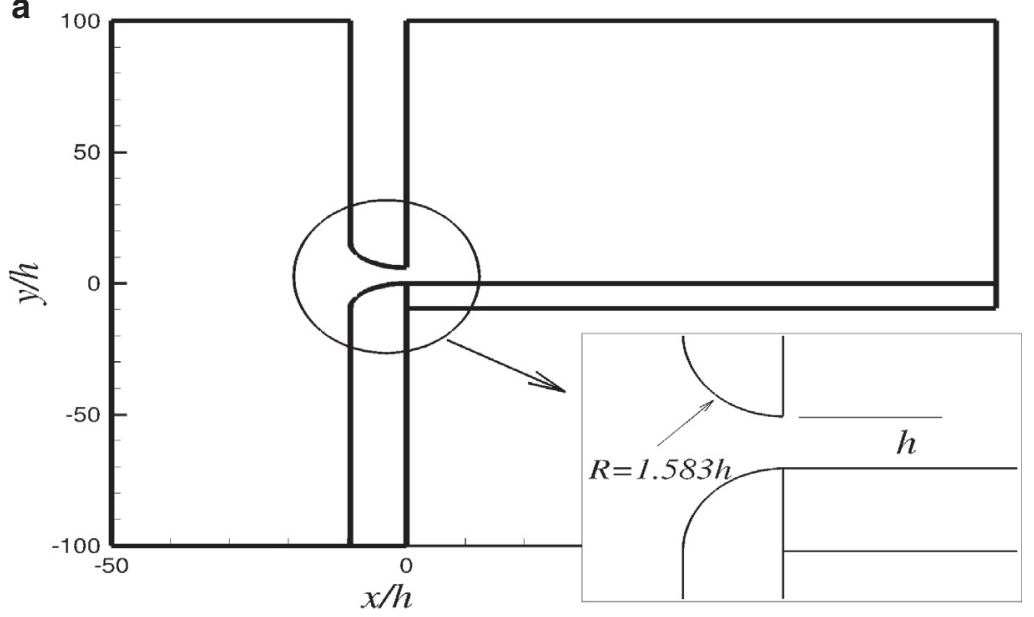

b

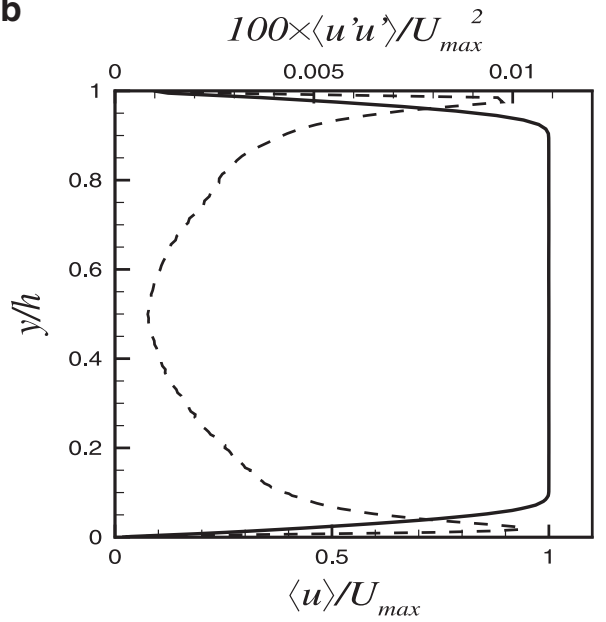

Fig. 2. (a) Schematic of the inlet nozzle from the experiment (Rostamy et al., 2011). (b) The inlet profiles for the mean streamwise velocity ( ) and the Reynolds stress $(---)$.
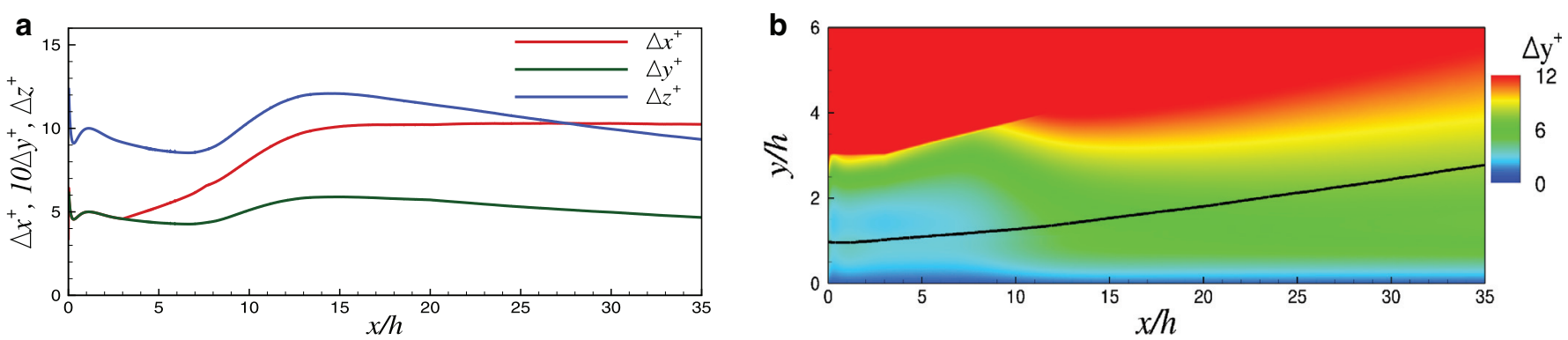

Fig. 3. (a) Grid spacing in wall units along the streamwise direction. (b) The distribution of $\Delta y^{+}$in the flow region. The solid line gives the location of $y=y_{1 / 2}$.
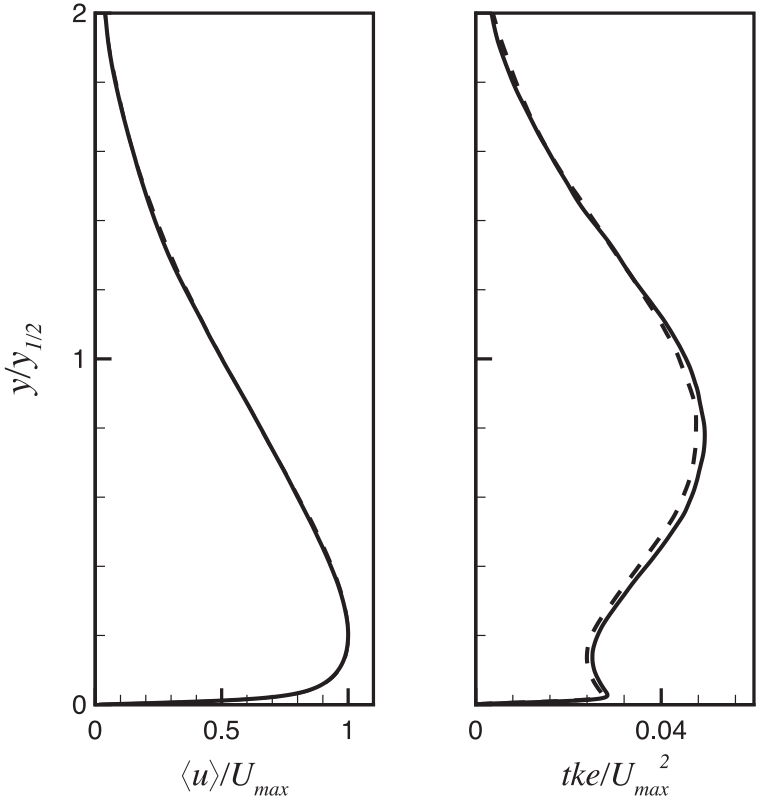

Fig. 4. Comparison of fine (__ $)$ and coarse $(---)$ grid for mean streamwise velocity $\langle u\rangle$ and turbulent kinetic energy tke $=0.5\left(\left\langle u^{\prime} u^{\prime}\right\rangle+\left\langle v^{\prime} v^{\prime}\right\rangle+\left\langle w^{\prime} w^{\prime}\right\rangle\right)$.

The simulation is also performed on a coarse grid with $1250 \times 344 \times 194$ grid points, totalling 83 million cells. Fig. 4 compares the mean streamwise velocity and turbulence kinetic energy at $x / h=30.0$ for the two grids. There is no significant difference between the mean flow for the two grids. The turbulent kinetic en- ergy shows a maximum of $4 \%$ difference. All the results presented in this work are for the fine grid.

\section{Results and discussions}

The main focus of this work is to present the heat transfer properties of the wall jet. However, the mean flow parameters are also included to assess the quality of the underlying flow field.

\subsection{Mean flow properties}

Fig. 5 shows the profiles for mean streamwise velocity $\langle u\rangle / U_{\max }$, Reynolds normal stresses $\left\langle u^{\prime} u^{\prime}\right\rangle / U_{\max }^{2},\left\langle v^{\prime} v^{\prime}\right\rangle / U_{\max }^{2}$ and Reynolds shear stress $\left\langle u^{\prime} v^{\prime}\right\rangle / U_{\max }^{2}$ at $x / h=30.0$. $U_{\max }$ is the local maximum streamwise velocity and \langle\rangle represents time averaging. The current DNS results are compared with an LES study Banyassady and Piomelli (2014) at the same Reynolds number and two different experiments at $R e=7500$ (Rostamy et al., 2011) and $R e=9700$ (Eriksson et al., 1998). The mean streamwise velocity profile from the DNS matches well with the LES (Banyassady and Piomelli, 2014) and the experiment (Rostamy et al., 2011). The Reynolds normal and shear stresses for the experiment at $R e=7500$ (Rostamy et al., 2011) have a higher overall level than the current DNS, the LES of (Banyassady and Piomelli, 2014) and experimental values at $R e=9700$ (Eriksson et al., 1998). However, the current DNS has the same level of Reynolds stresses as the LES of (Banyassady and Piomelli, 2014) and the experimental values at $R e=9700$ (Eriksson et al., 1998). Banyassady and Piomelli (2014) used a time dependent fully developed turbulent channel flow field at $R e=7500$ as an inflow boundary condition in their LES. They also used scaled Reynolds stress profiles from the experiment (Rostamy et al., 2011) 


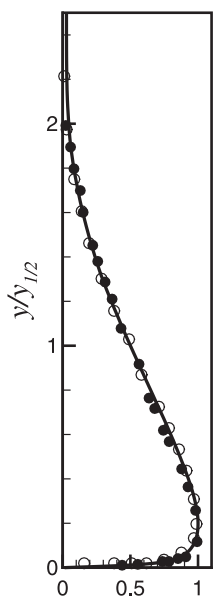

$\langle u\rangle / U_{\max }$
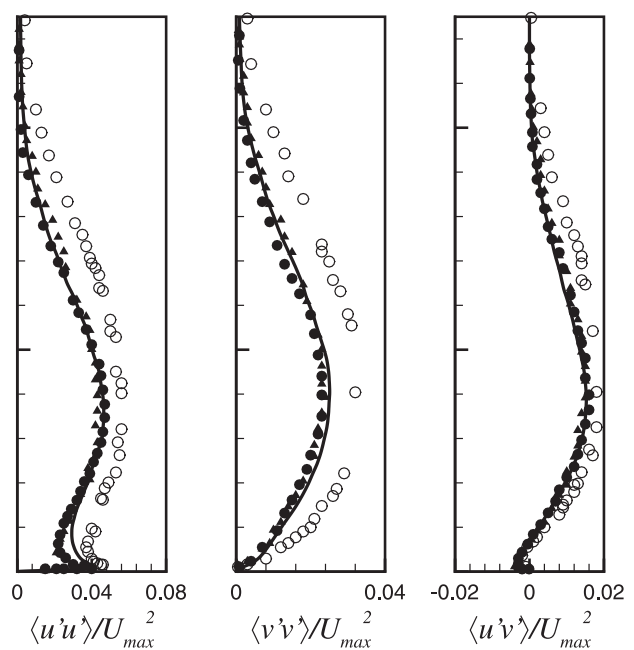

Fig. 5. Profiles of mean streamwise velocity, mean streamwise Reynolds stress, mean wall-normal Reynolds stress and mean Reynolds shear stress at $x=30.0 h$ : Current DNS

), (Banyassady and Piomelli, 2014) (•), (Rostamy et al., 2011) (०) and (Eriksson et al., 1998) ( $\mathbf{\Lambda}$ ).

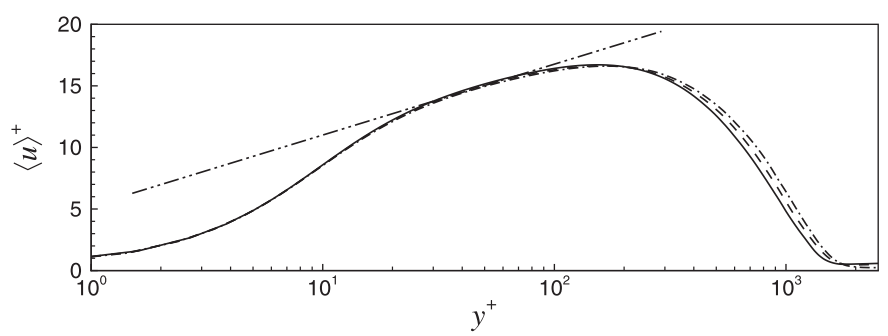

Fig. 6. Inner scaled mean velocity profiles: Current DNS $x=25.0 h(\longrightarrow), x=$ 30.0h (- - ), $x=35.0 h$ ( - - —); $\log -\operatorname{law} \frac{1}{0.40} \ln y^{+}+5.2$ (_ - —).

to force the flow at $x / h=2.0,4.0,6.0$ and 8.0. However, with the fully developed turbulent inflow and forcing, their Reynolds stress levels are still lower than the experiment for $R e=7500$ (Rostamy et al., 2011). The current DNS relies almost entirely on natural shear layer and wall transition for turbulence development. Despite this, it reaches the same turbulence level as the LES (Banyassady and Piomelli, 2014).

Fig. 6 shows the inner scaled mean streamwise velocity $\langle u\rangle^{+}$ profiles at different streamwise locations of $x / h=25.0,30.0$ and 35.0. The profiles give good collapse in the near wall region up to $y^{+}=200.0$. The inner scaled profiles are also in agreement with the log-law, $\langle u\rangle^{+}=\frac{1}{\kappa} \ln y^{+}+A$ with $\kappa=0.4$ and $A=5.2$, from $y^{+}=30.0$ to 90.0 . The current values are close to the reported values of $\kappa=0.41$ and $A=5.0$ in several other studies, for example, see Abrahamsson et al. (1994); Dejoan and Leschziner (2005); Eriksson et al. (1998).

The growth rate of the wall jet is measured as the streamwise variation of the jet half width $y_{1 / 2}$. Fig. $7(a)$ shows that the variation of $y_{1 / 2}$ from the DNS is in agreement with the linear relationship $0.0732 x / h+0.332$ proposed by Abrahamsson et al. (1994). However, George et al. (2000) have argued that $d y_{1 / 2} / d x$ is dependent on $x$. The Reynolds number and the streamwise distance considered here are not large enough to show such dependence. The variation of the maximum local velocity $U_{\max }$ and the wall shear stress $\tau_{w}$ are compared with the LES (Banyassady and Piomelli, 2014) in Fig. 7(b) and (c), respectively. The predicted $\tau_{w}$ is close to the LES (Banyassady and Piomelli, 2014), whereas $U_{\max }$ has a slower decay rate. The difference might be due to the different conditions employed for the entrainment in current DNS and LES (Banyassady and Piomelli, 2014).
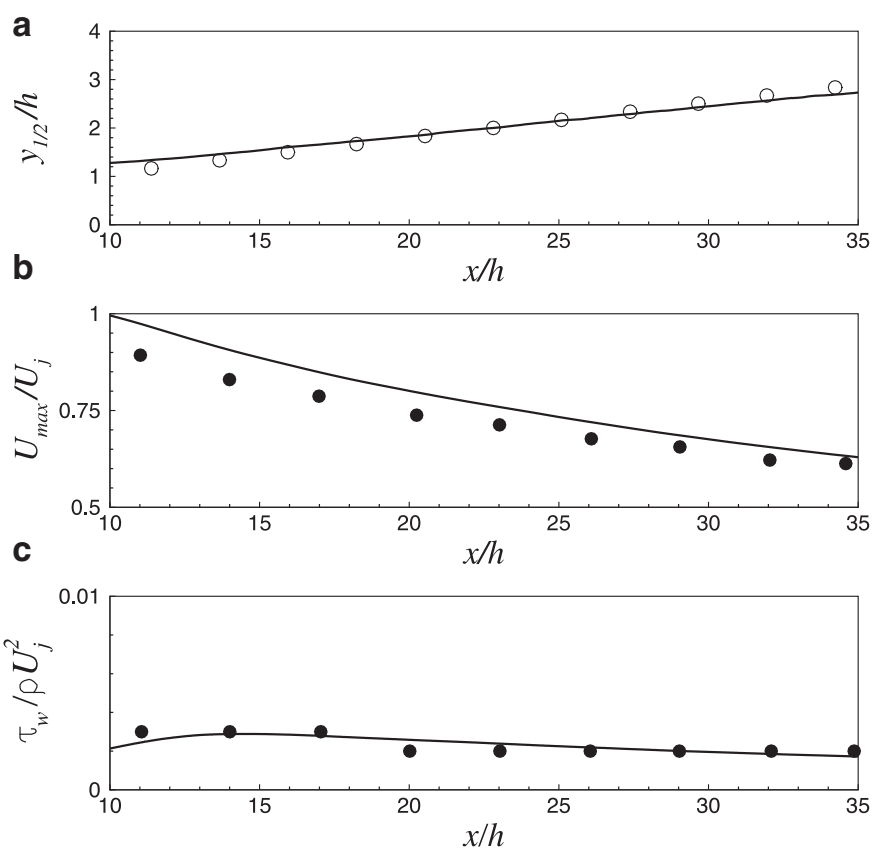

Fig. 7. (a) Jet half width, $0.0732 x / h+0.332$ (o), (b) maximum local velocity and (c) wall shear stress : Current DNS (__ $)$; (Banyassady and Piomelli, 2014) (•).

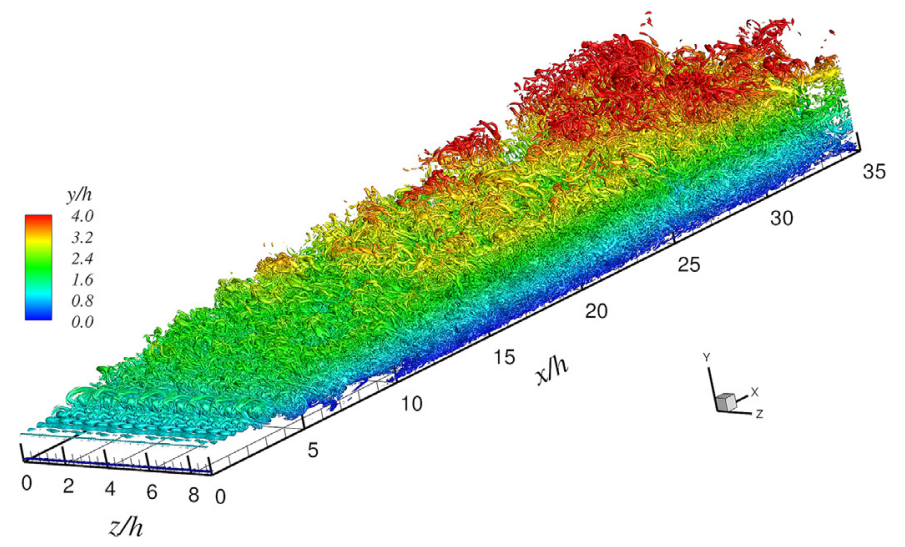

Fig. 8. Coherent vortical structures in the wall jet visualised by iso-surfaces of $Q=$ 0.9 . The iso-surfaces are coloured with wall normal distance $y / h$. (For interpretation of the references to colour in this figure legend, the reader is referred to the web version of this article.)

\subsection{Instantaneous flow and temperature field}

The instantaneous flow field provides an overall picture of the jet shear layer and its interaction with the wall. In this simulation several instantaneous fields are saved and visualised through iso-surfaces of the second-invariant of the velocity gradient tensor $Q=-\left(\partial u_{i} / \partial x_{j}\right)\left(\partial u_{j} / \partial x_{i}\right)$ (Hunt et al., 1988). Fig. 8 shows one such realisation of the wall jet indicating large scale vortical structures. The $Q$ iso-surfaces are coloured with the wall normal distance $y / h$. Initially roll structures are formed in the outer shear layer within $x / h<3.0$. They become unstable and streamwise structures are formed. As a result, large roll structures collapse downstream. Under the influence of passing shear layer structures, the near wall flow also transitions to turbulence. These near wall and shear layer structures develop strong interaction and mixing, which is responsible for momentum and heat transfer in the wall jet.

The effect of turbulence on the heat transport is shown by the iso-thermal surfaces at $T=0.50$ and 0.25 in Fig. 9. The 


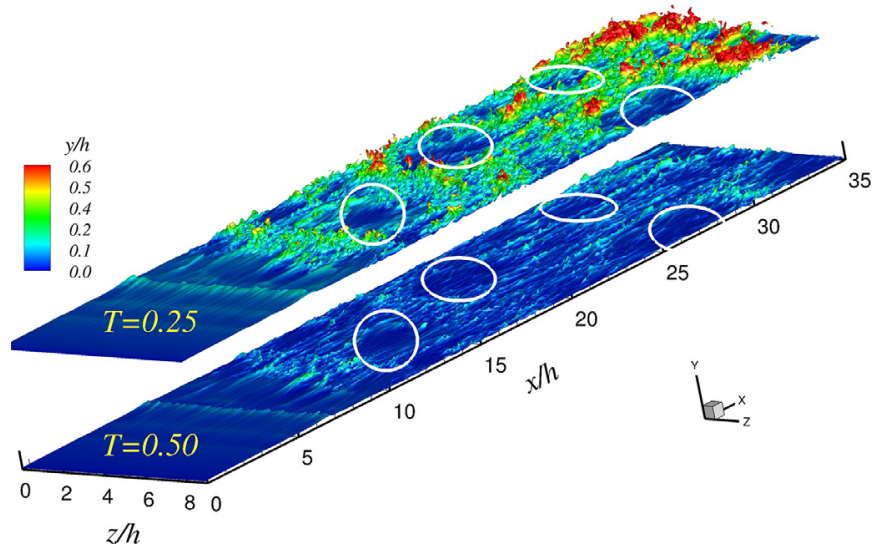

Fig. 9. Iso-thermal surfaces at $T=0.25$ and 0.50 are coloured with wall normal height $y / h$. The surface $T=0.25$ is shifted vertically upward by $y / h=4.5$. The low penetration spots are marked with white circles. (For interpretation of the references to colour in this figure legend, the reader is referred to the web version of this article.)
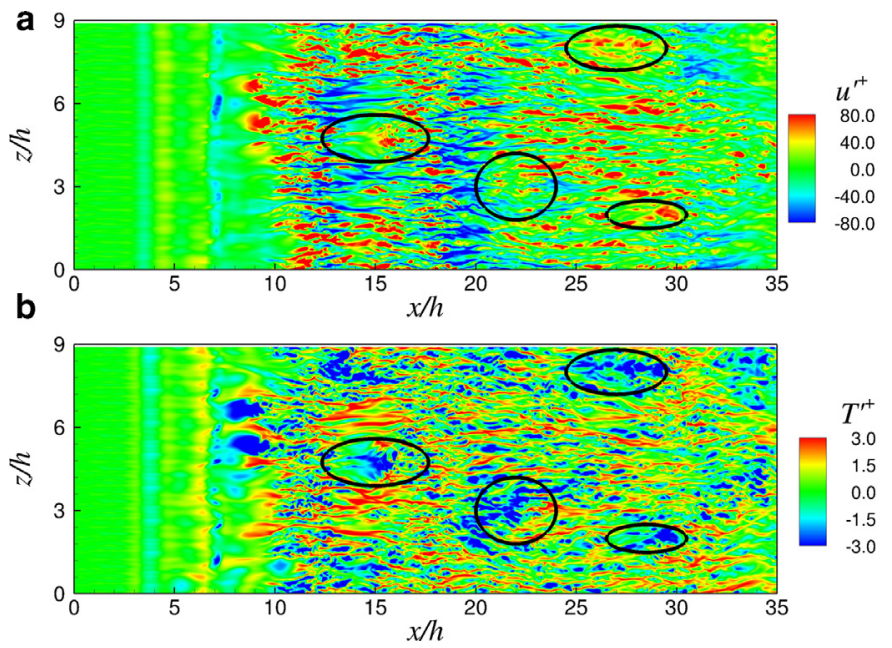

Fig. 10. Instantaneous fluctuating flow and temperature fields at $y^{+} \approx 7$, (a) $u^{\prime+}$ and (b) $T^{\prime+}$. Few high velocity and corresponding low temperature patches are marked on the contours.

surface at $T=0.25$ is shifted upward in the wall normal direction for the clarity. The surfaces are coloured with the wall normal distance $y / h$ and represent the same instant as in Fig. 8. Once the flow undergoes transition, the iso-thermal surface area starts to increase, which indicates enhanced mixing and transport. This mixing and transport of heat away from the wall, increases farther downstream. The heat transport away from the wall is inhomogeneous. There are certain low penetration spots which are marked on the iso-thermal surfaces.

Fig. 10 shows the contours of instantaneous streamwise velocity fluctuations $u^{\prime+}=(u-\langle u\rangle) / u_{\tau}$ and temperature fluctuations $T^{\prime+}=\frac{\rho u_{\tau} c_{p}(T-\langle T\rangle)}{q_{w}}$ at $y^{+} \approx 7$ corresponding to the same instant in Figs. 8 and 9 . Here, $u_{\tau}$ is the local friction velocity, $\rho$ is the density, $c_{p}$ is the specific heat capacity of the fluid, $q_{w}=-\left.k \frac{\partial T}{\partial y}\right|_{y=0}$ is the heat transfer from the wall to the fluid and $k$ is the thermal conductivity of the fluid. The $u^{\prime+}$ contours show low velocity streaks, however they are different from fully developed boundary layer structures presented in other studies, see, for example Kline et al. (1967); Li et al. (2009) and Kong et al. (2000). In a fully developed boundary layer, low velocity streaks are separated by wider high velocity zones in the spanwise direction. In the current wall jet simulation, low velocity streaks are not regularly spaced
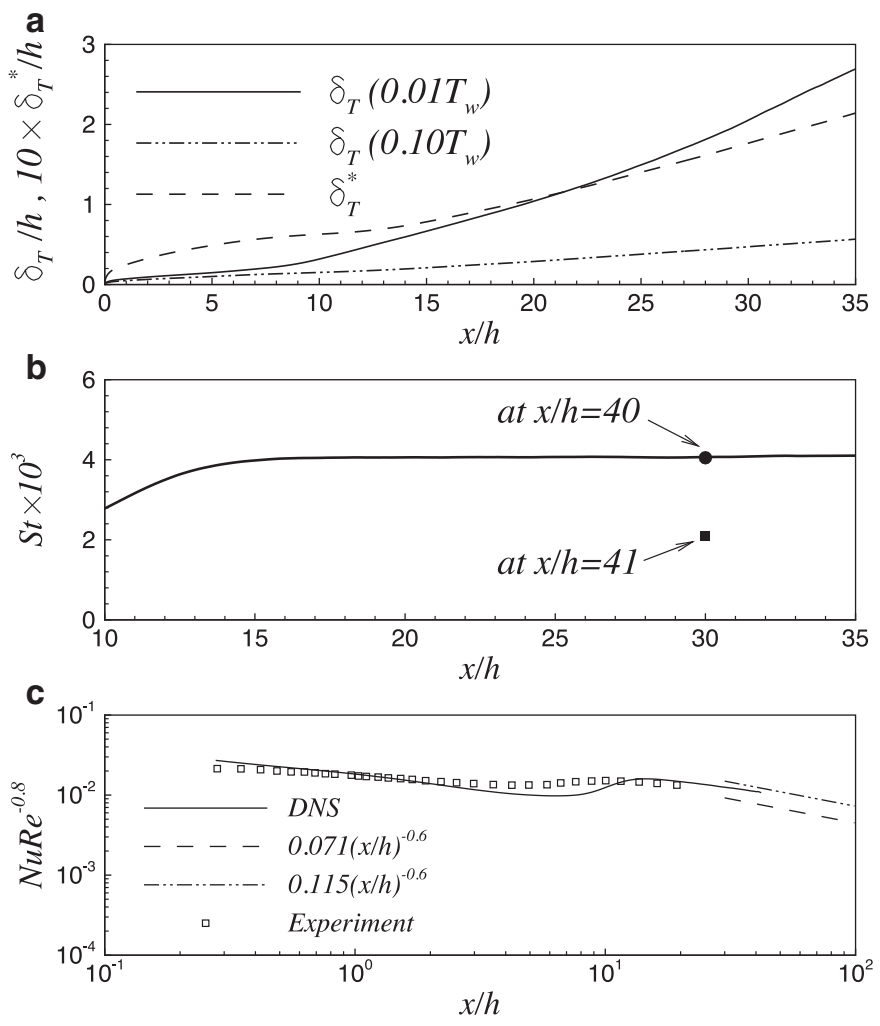

Fig. 11. The streamwise development of (a) Thermal boundary layer $\delta_{T}$ and thermal displacement thickness $\delta_{T}^{*}$, (b) Stanton number (St) : Current DNS (_ Experiment (Dacos et al., 1984) (ם), (Nizou, 1981) (•) and (c) Nusselt number Nu: Current DNS ( $\_$); Experiment (AbdulNour et al., 2000) $(\square)$; $0.071(x / h)^{-0.6}$

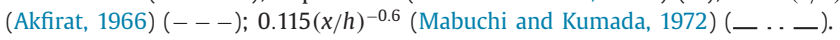

with high velocity zones. However, the character of low velocity streaks in terms of heat transfer is similar to a turbulent boundary layer. The low speed streaks coincide with high temperature streaks, whereas high speed streaks coincide with low temperature streaks (Iritani et al., 1985). The low speed streaks form near the wall, which convect away the heat. The high speed streaks transport cold fluid from the fast moving outer flow. Several cold patches and corresponding high velocity zones are marked on the contours in Fig. 10, which are coincident with the low penetration zones in Fig. 9.

\subsection{Mean heat transfer properties}

The development of the thermal boundary layer is shown in Fig. 11(a). The thermal boundary layer thickness $\delta_{T} / h$ identifies the outer edge. It is defined as a distance from the wall, where an arbitrary value of the temperature is achieved. Two different values of temperature $T=0.10 T_{w}$ and $0.01 T_{w}$ are considered. The arbitrary nature of these definitions is reflected by the two substantially different profiles presented. The thermal boundary layer shows a slow growth up to $x / h<8.0$. After the transition it grows at a faster rate. Fig. 11(a) also shows the variation of thermal displacement thickness $\delta_{T}^{*}=\int_{0}^{\infty} \frac{T-T_{\infty}}{T_{w_{w}}-T_{\infty}} d y$, which is free from any arbitrary definition. The thermal displacement thickness shows a linear growth in the fully developed region $(x / h>20.0)$ of the wall jet.

Fig. 11 (b) shows the variation of the Stanton number St, which is defined as;

$S t=\frac{h_{c}}{\rho U_{\max } c_{p}}=\frac{N u}{\operatorname{RePr}}=\frac{q_{w}}{\rho U_{\max } c_{p}\left(T_{w}-T_{\infty}\right)}$,

where $h_{c}$ is the convection heat transfer coefficient and $N u$ is the Nusselt number. The Stanton number provides a measure of the 

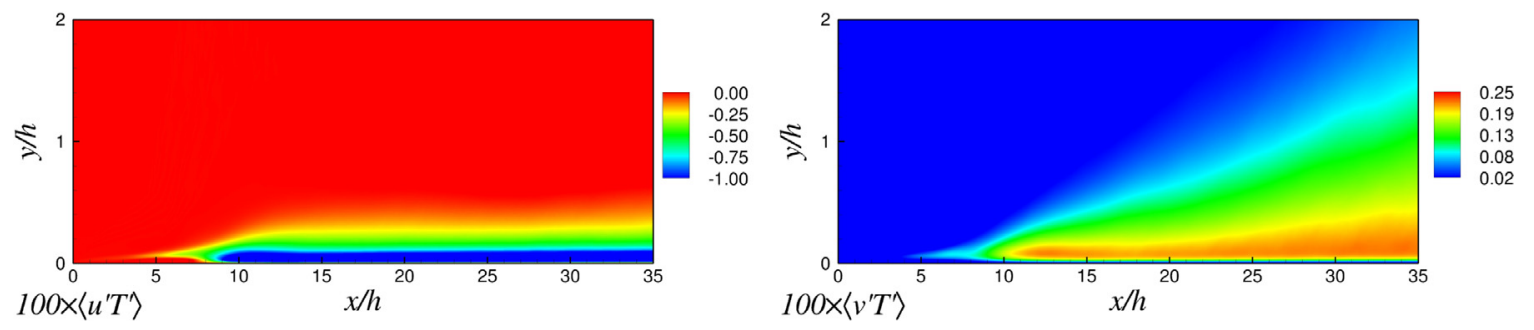

Fig. 12. Contours of turbulent heat flux components in the streamwise direction $\left\langle u^{\prime} T^{\prime}\right\rangle$ and the wall-normal direction $\left\langle v^{\prime} T^{\prime}\right\rangle$.

ratio of the heat transferred to the fluid relative to its heat capacity. It also relates the wall shear stress to the total heat transfer at the wall. The current DNS gives almost a constant St value of $4.0 \times 10^{-3}$ in the fully developed region, which is compared to the measured values reported by Nizou (1981) for a plane wall jet $(R e=9000)$ and Dacos et al. (1984) for a wall jet with an external stream at $R e=30,000$. The DNS is in good agreement with the plane wall jet measurements (Nizou, 1981).

Fig. 11 (c) shows the variation of Nusselt number $N u$, which gives the ratio of convective to conductive heat transfer at the wall. It is defined as;

$N u=\frac{h_{c} L}{k}=\frac{\left.\frac{\partial\left(T_{w}-T\right)}{\partial y}\right|_{y=0}}{\frac{\left(T_{w}-T_{\infty}\right)}{L}}$,

where $L$ is the characteristic length scale of the flow, here taken as the jet height, $h$. The Nusselt number coefficient is presented in a non-dimensional form $N u R e^{-0.8}$ and compared with the experimental data of AbdulNour et al. (2000) for a wall jet at $R e=$ 7700. The experimental measurements are only available up to $x / h=20.0$. At fully developed downstream locations $x / h>30.0$, the Nusselt number coefficient can be related to the streamwise distance as $N u R e^{-0.8}=C(x / h)^{-0.6}$, where $C$ is a numerical coefficient. Empirical data suggests $C$ has a range from 0.071 (Akfirat, 1966 ) to 0.115 (Mabuchi and Kumada, 1972). However, these correlations are only valid for $x / h>30.0$. The relation given by the current DNS lies within the two constants, yet is closer to Mabuchi and Kumada (1972).

The turbulent heat flux shown in Fig. 12 is a major component of heat transfer from the wall to the outer flow. As shown in Fig. 12(a), the streamwise component $\left\langle u^{\prime} T^{\prime}\right\rangle$ of the heat flux is negative near the wall. The production of $\left\langle u^{\prime} T^{\prime}\right\rangle$ depends on $\mathscr{P}_{\left\langle u^{\prime} T^{\prime}\right\rangle} \sim-\left\langle u^{\prime} v^{\prime}\right\rangle \partial\langle T\rangle / \partial y$. With both shear stress and temperature gradient negative near the wall, the streamwise turbulent heat flux becomes negative. It transports heat against the direction of the mean flow. The wall-normal heat flux component $\left\langle v^{\prime} T^{\prime}\right\rangle$ is positive and responsible for heat transfer away from the wall. The dominant term in the wall-normal turbulent flux production is $\mathscr{P}_{\left\langle v^{\prime} T^{\prime}\right\rangle} \sim-\left\langle v^{\prime} v^{\prime}\right\rangle \partial\langle T\rangle / \partial y$. Fig. 12(b) shows a high value of $\left\langle v^{\prime} T^{\prime}\right\rangle$ is generated near the wall due to a high negative temperature gradient.

The fluctuating streamwise velocities and temperature contours in Fig. 10 show that a high level of correlation exists in the streamwise velocity $u$ and temperature $T$. The correlation can be quantified through a correlation coefficient $\rho_{u T}=\frac{\left\langle u^{\prime} T^{\prime}\right\rangle}{u_{r m s} T_{r m s}}$, where the root mean square $(r m s)$ values are $u_{r m s}=\sqrt{\left\langle u^{\prime} u^{\prime}\right\rangle}$ and $T_{r m s}=\sqrt{\left\langle T^{\prime} T^{\prime}\right\rangle}$. Fig. 13 shows the near wall variation of correlation coefficient. The current DNS results are compared with fully developed turbulent boundary layer (Antonia et al., 1988) and fully developed pipe (Bremhorst and Bullock, 1970) flows. Antonia et al. (1988) have suggested that $\rho_{u T}$ approaches unity at the wall. It is difficult to measure temperature and velocity close to the wall and other studies (Bremhorst and Bullock, 1970; Wardana et al., 1995) resulted in a value less than one. The current DNS also indicates values of $\rho_{u T}$

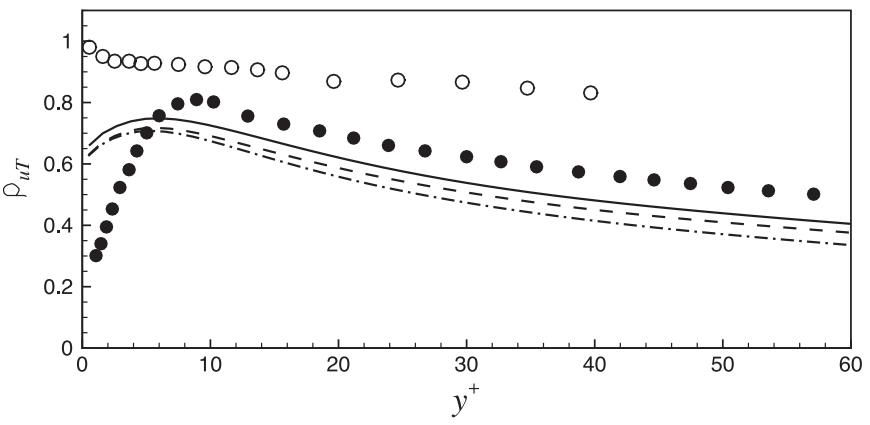

Fig. 13. The correlation coefficient $\rho_{u T}$ in the near wall region: Current DNS at $x=$ $25.0 h$ (— $), x=30.0 h(---), x=35.0 h$ ( $-\cdots)$; boundary layer by Antonia et al. (1988) (०); pipe flow by Bremhorst and Bullock (1970) (•).

less than one. After peaking at $y^{+} \approx 6$, it decreases with increasing distance downstream.

\subsection{Turbulent Prandtl number}

The turbulent Prandtl number $P r_{t}$ is an important parameter for heat transfer. It is defined as the ratio of the turbulent eddy viscosity $v_{t}$ and eddy diffusivity $\alpha_{t}$ i.e. $P r_{t}=v_{t} / \alpha_{t}$, where

$v_{t}=-\frac{\left\langle u^{\prime} v^{\prime}\right\rangle}{\frac{\partial\langle u\rangle}{\partial y}}$ and $\alpha_{t}=-\frac{\left\langle v^{\prime} T^{\prime}\right\rangle}{\frac{\partial\langle T\rangle}{\partial y}}$.

For many flows $\operatorname{Pr}_{t}$ is considered as a constant value. It is used to evaluate $\alpha_{t}$ from $v_{t}$, for heat transfer calculations. In the case of turbulent boundary layers with isothermal boundary conditions, $P r_{t}$ reaches $\sim 1.1$ ( $\mathrm{Li}$ et al., 2009) in the near wall region. It is well known that for the wall jet, $v_{t}$ becomes negative before reaching $y=y_{\max }$ and the Boussinesq approximation is not valid (Launder and Rodi, 1983). Fig. 14 shows $\operatorname{Pr}_{t}$ profiles plotted against $y / y_{1 / 2}$, $y / y_{\theta / 2}$ and $y^{+} . \operatorname{Pr}_{t}$ is not a constant for this flow. It is higher than 1.0 below $y^{+} \approx 1$, constant in the viscous sublayer $\left(y^{+}<10.0\right)$ and decays rapidly on moving away from it. $\operatorname{Pr}_{t}$ becomes negative before $y=y_{\max }$. At $y=y_{\max }, \partial\langle u\rangle / \partial y=0.0$, which makes $v_{t}$ and $\operatorname{Pr}_{t}$ infinite. This is reflected in the discontinuity in Fig. 14(a) and (b) at $y / y_{1 / 2}=0.2$ and $y / y_{\theta / 2}=12$, respectively.

\subsection{Scaling of heat transfer parameters}

Fig. 15 shows the outer scaled, thermal scaled and inner scaled mean temperature $\langle T\rangle$ profiles at $x / h=25.0,30.0$ and 35.0. The inner scaled temperature $\langle T\rangle^{+}$is defined as;

$\langle T\rangle^{+}=\frac{\left(T_{w}-T\right)}{T_{\tau}} \quad$ and $\quad T_{\tau}=\frac{q_{w}}{\rho u_{\tau} c_{p}}$,

where $T_{\tau}$ is the friction temperature.

The mean temperature profiles show good scaling behaviour for all the three scaling parameters. The temperature profiles are compared with the experimental results of Dacos et al. (1984), for a 
a

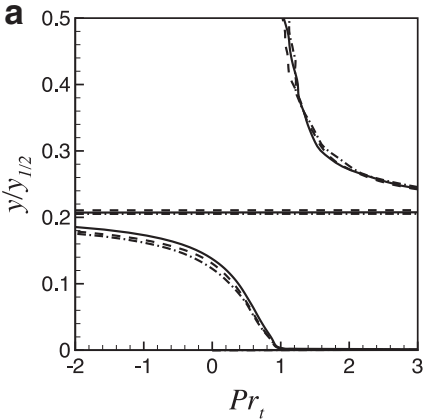

C

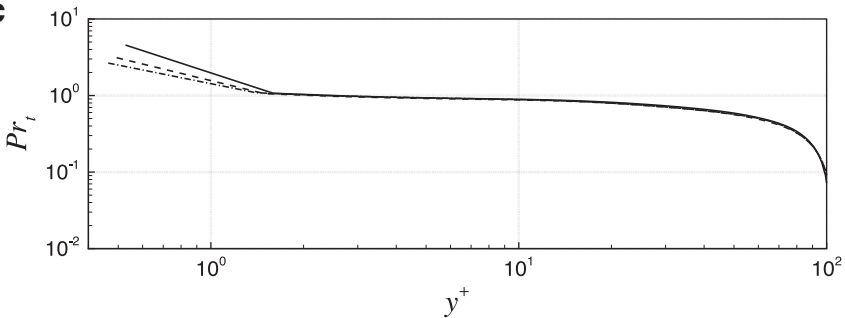

Fig. 14. The profile of turbulent Prandtl number against (a) outer variables $y / y_{1 / 2}$, (b) $y / y_{\theta / 2}$ and (c) inner variable $y^{+}$at $x=25.0 h$ (_ $), x=30.0 h(---), x=$ $35.0 h(-\cdots)$
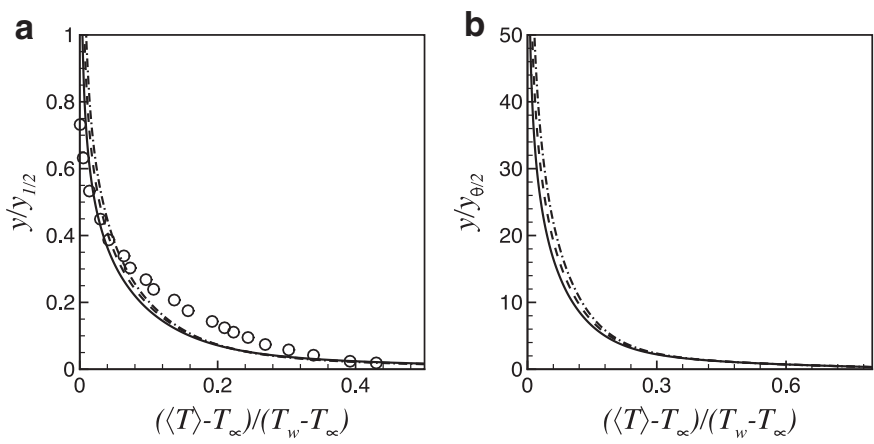

C

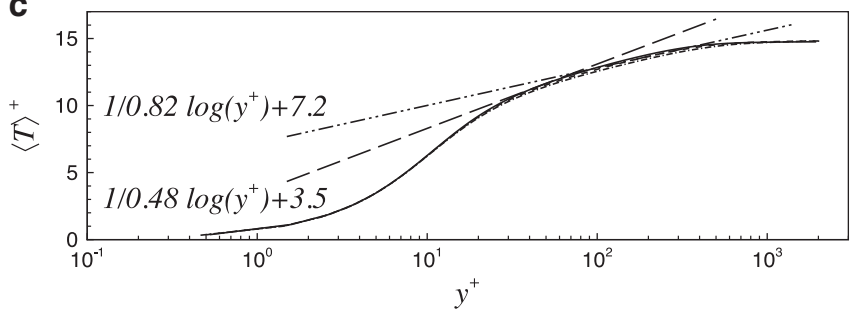

Fig. 15. Mean temperature profiles scaled with (a) outer variables $y / y_{1 / 2}$, (b) ther-

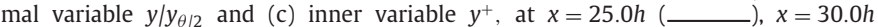
$(---)$ and $x=35.0 h$ ( - - - ). (Dacos et al., 1984) (o).

wall jet with an external stream at $R e=30,000$. There is agreement between the current DNS and the experiment. Several studies have defined a log-law profile for the inner scaled temperature as $\frac{1}{\kappa_{\theta}} \ln y^{+}+A_{\theta}$. Such a log-law based on the current DNS, with $\kappa_{\theta}=0.48$ and $A_{\theta}=3.5$ is shown in Fig. 15(c), which are comparable to $\kappa_{\theta}=0.48$ and $A_{\theta}=3.8$ recommended by Kader and Yaglom (1972). This log-law is valid for a flat plate zero-pressure gradient thermal boundary layer with $\operatorname{Pr}=0.7$. Another log-law with $\kappa_{\theta}=0.82$ and $A_{\theta}=7.2$ is also included in Fig. 15(c), which has the same slope as suggested by Nizou (1981) for a plane wall jet with a Prandtl number $P r=0.7$ and Reynolds number $R e=14,400$. This log-law comes close to the DNS only in the range of $y^{+}>80.0$, which is beyond the normal log-law region for wall jets. Dacos et al. (1984) have pointed out that the log-law of Nizou (1981) for temperature is related to a velocity profile with $\kappa=0.55$ and $A=$ 8.1, which is far from the accepted boundary layer form.
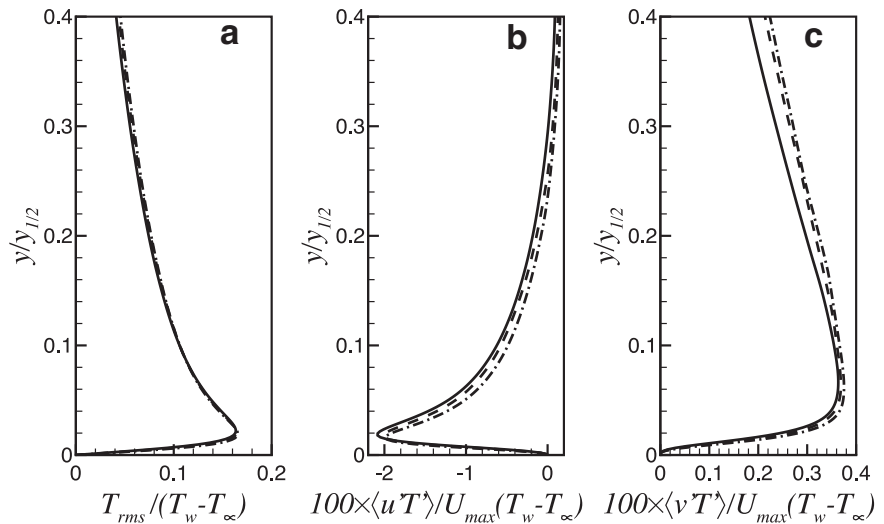

Fig. 16. The outer scaled profiles of (a) $T_{r m s}$, (b) $\left\langle u^{\prime} T^{\prime}\right\rangle$ and (c) $\left\langle v^{\prime} T^{\prime}\right\rangle$, at $x=25.0 h$ ), $x=30.0 h(---)$ and $x=35.0 h(\ldots-\ldots)$.
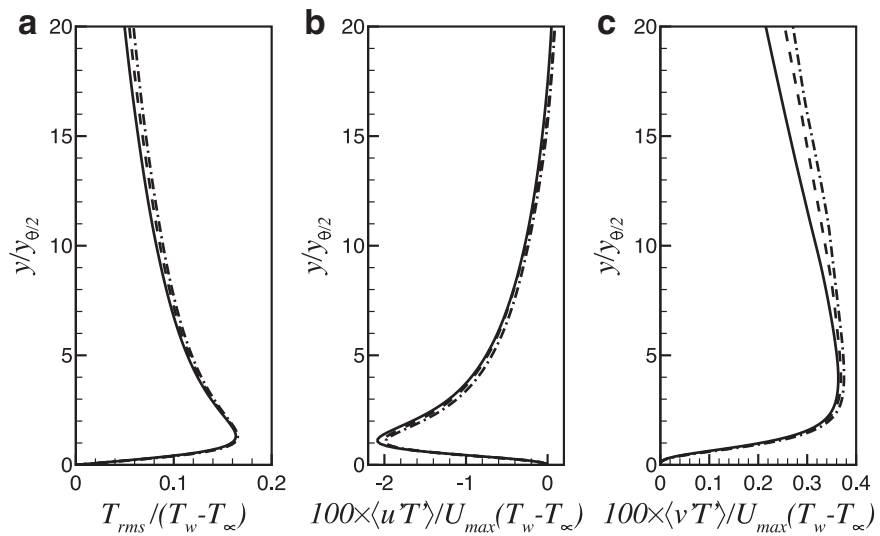

Fig. 17. The profiles scaled with $y / y_{\theta / 2}$; (a) $T_{r m s}$, (b) $\left\langle u^{\prime} T^{\prime}\right\rangle$ and (c) $\left\langle v^{\prime} T^{\prime}\right\rangle$, at $x=25.0 \mathrm{~h}$ ), $x=30.0 h(--)$ and $x=35.0 h(-\ldots)$.

Figs. 16-18 show the temperature root mean square $T_{r m s}$ and turbulent heat fluxes $\left(\left\langle u^{\prime} T^{\prime}\right\rangle\right.$ and $\left.\left\langle v^{\prime} T^{\prime}\right\rangle\right)$, scaled with the outer variable, the thermal variable and the inner variable, respectively. The $T_{r m s}$ and $\left\langle u^{\prime} T^{\prime}\right\rangle$ show good scaling behaviour for both the outer and thermal variables. The profiles for wall normal heat flux $\left\langle v^{\prime} T^{\prime}\right\rangle$ are similar to each other at downstream locations of $x / h=30.0$ and 35.0 with both outer and thermal scaling. In the near wall region, $T_{r m s}$ and turbulent heat fluxes scale with the inner variable, which are given as $T_{r m s}^{+}=\frac{T_{r m s}}{T_{\tau}},\left\langle u^{\prime} T^{\prime}\right\rangle^{+}=\frac{\left\langle u^{\prime} T^{\prime}\right\rangle}{u_{\tau} T_{\tau}}$ and $\left\langle v^{\prime} T^{\prime}\right\rangle^{+}=\frac{\left\langle v^{\prime} T^{\prime}\right\rangle}{u_{\tau} T_{\tau}}$.

\subsection{Temperature variance and heat flux budgets}

The budget for the temperature variance $\left\langle T^{\prime} T^{\prime}\right\rangle$ is given as,

$\mathscr{C}_{\left\langle T^{\prime} T^{\prime}\right\rangle}=\mathscr{P}_{\left\langle T^{\prime} T^{\prime}\right\rangle}+\varepsilon_{\left\langle T^{\prime} T^{\prime}\right\rangle}+\mathscr{T}_{\left\langle T^{\prime} T^{\prime}\right\rangle}+\mathscr{D}_{\left\langle T^{\prime} T^{\prime}\right\rangle}$

where

$$
\begin{array}{lr}
\mathscr{C}_{\left\langle T^{\prime} T^{\prime}\right\rangle}=\left\langle u_{i}\right\rangle \frac{\partial\left\langle T^{\prime} T^{\prime}\right\rangle}{\partial x_{i}} & \text { Convection } \\
\mathscr{P}_{\left\langle T^{\prime} T^{\prime}\right\rangle}=-2\left\langle u_{i}^{\prime} T^{\prime}\right\rangle \frac{\partial\langle T\rangle}{\partial x_{i}} & \text { Production } \\
\varepsilon_{\left\langle T^{\prime} T^{\prime}\right\rangle}=-\frac{2}{\operatorname{RePr}}\left\langle\left(\frac{\partial T^{\prime}}{\partial x_{i}}\right)^{2}\right\rangle & \text { Dissipation } \\
\mathscr{T}_{\left\langle T^{\prime} T^{\prime}\right\rangle}=-\frac{\partial\left\langle u_{i}^{\prime} T^{\prime} T^{\prime}\right\rangle}{\partial x_{i}} & \text { Turbulent diffusion } \\
\mathscr{D}_{\left\langle T^{\prime} T^{\prime}\right\rangle}=\frac{1}{\operatorname{RePr} \frac{\partial^{2}\left\langle T^{\prime} T^{\prime}\right\rangle}{\partial x_{i}^{2}}} & \text { Molecular diffusion }
\end{array}
$$

Fig. 19 shows all the terms for the temperature variance $\left\langle T^{\prime} T^{\prime}\right\rangle$ budget, where all the terms are explicitly evaluated. The budget 

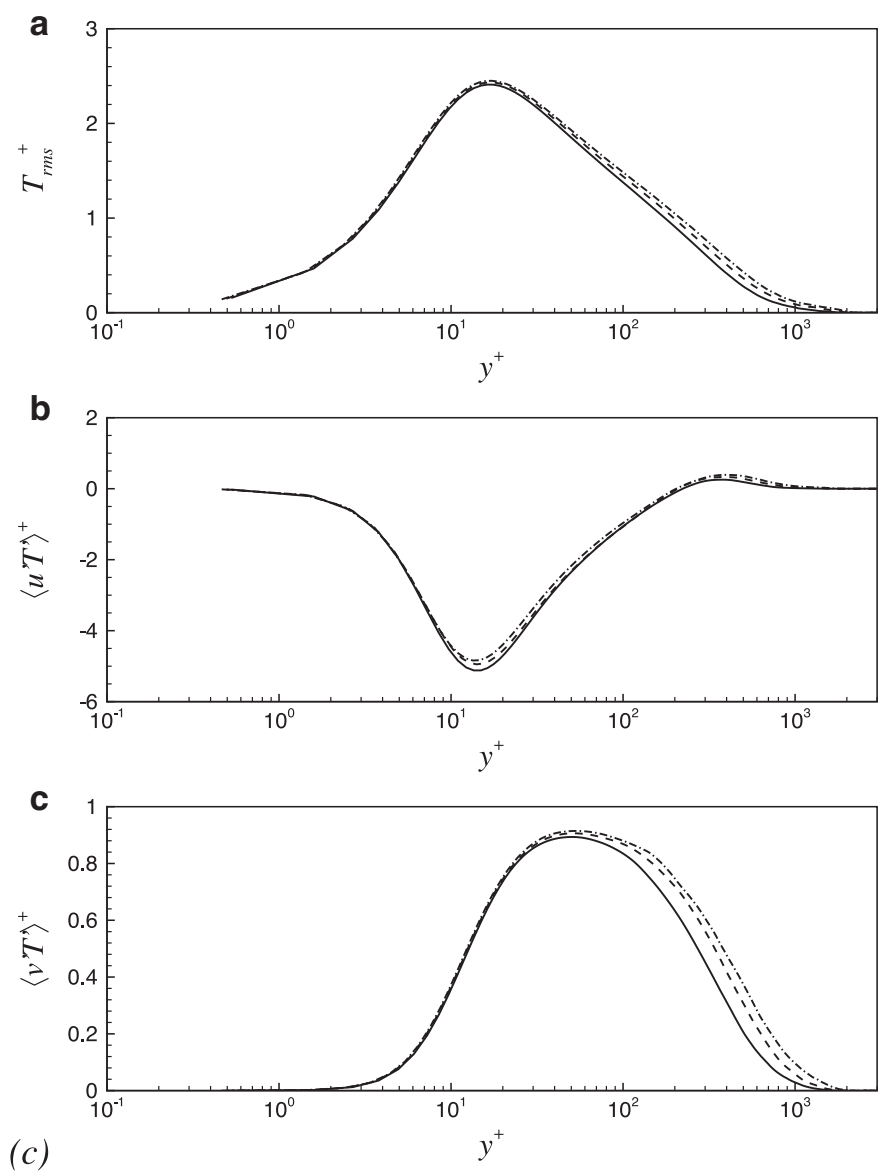

Fig. 18. The inner scaled profiles of (a) $T_{\text {rms }}^{+}$, (b) $\left\langle u^{\prime} T^{\prime}\right\rangle^{+}$and (c) $\left\langle v^{\prime} T^{\prime}\right\rangle^{+}$, at $x=25.0 h$ ), $x=30.0 h(---)$ and $x=35.0 h(-\cdot-)$.

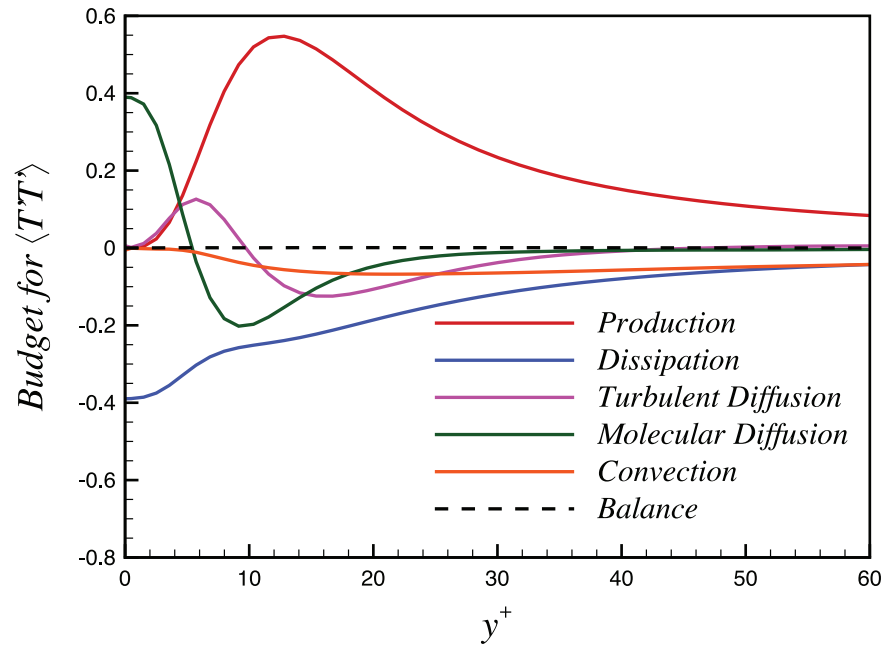

Fig. 19. The inner scaled budget of $\left\langle T^{\prime} T^{\prime}\right\rangle$ at $x / h=30.0$.

terms are scaled with the inner variables parameter $\frac{u_{\tau}^{3} T_{\tau}}{v}$. The balance for all the terms is also included, which is $O\left(10^{-3}\right)^{v}$. In the viscous sub layer, for $y^{+}<5$, the convection term is negligible. In this region, molecular diffusion is important, which balances the high dissipation close to the wall. The highest production level is around $y^{+}=10.0$, which coincides with the highest level of $T_{r m s}^{+}$(Fig. 18). This high production in the near wall region is due to a high negative temperature gradient $\partial\langle T\rangle / \partial y$ and high negative turbulent heat flux $\left\langle u^{\prime} T^{\prime}\right\rangle$. The high levels of production, up to $y^{+}=20.0$, are balanced by all the other terms of the budget. The production is small
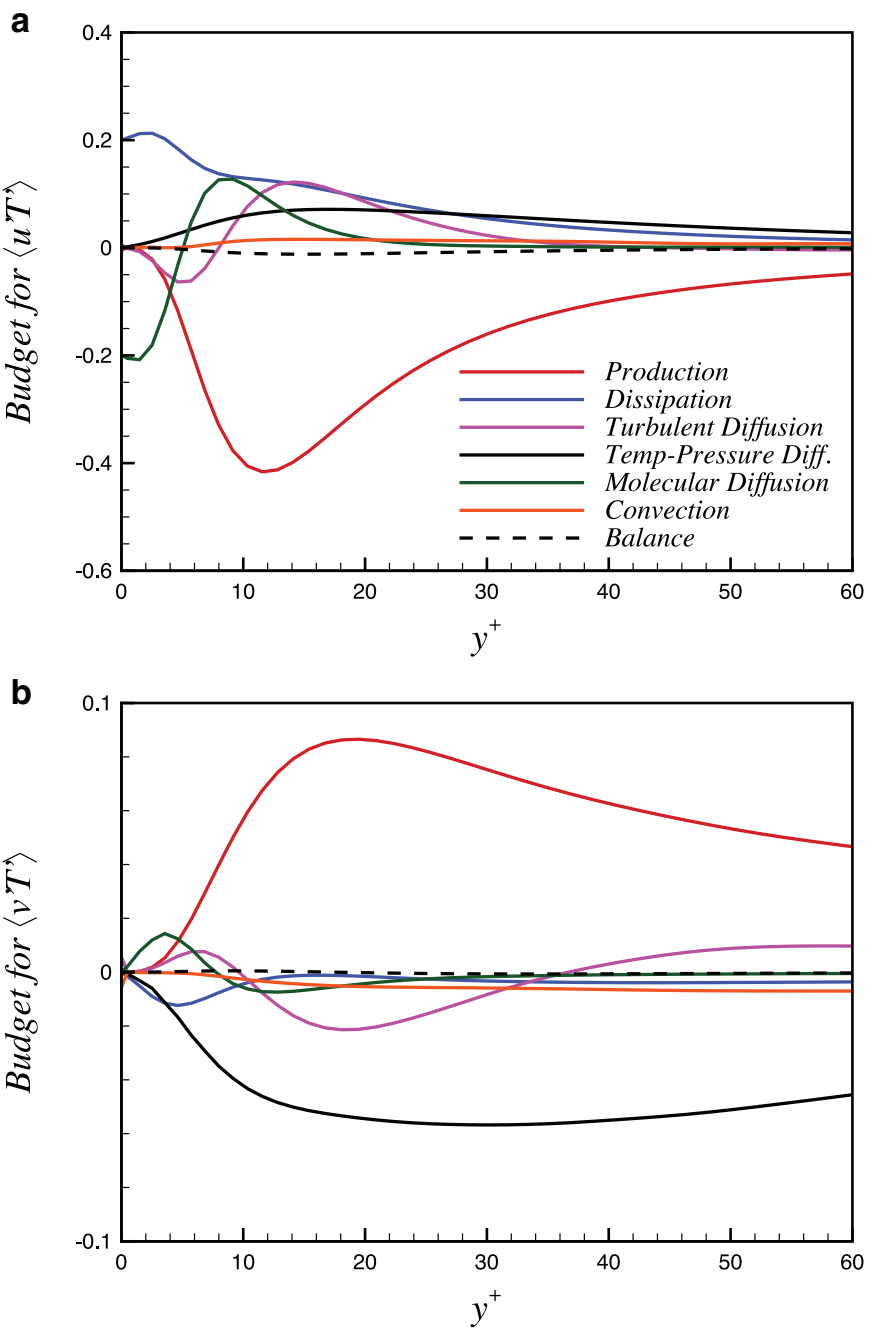

Fig. 20. The inner scaled budgets of (a) $\left\langle u^{\prime} T^{\prime}\right\rangle$ and (b) $\left\langle v^{\prime} T^{\prime}\right\rangle$ at $x / h=30.0$. The legend is same for the two budgets.

beyond $y^{+}=50.0$ and mainly balanced by dissipation and convection. In the near wall region $y^{+}<20$ turbulent diffusion is dominant in transporting $\left\langle T^{\prime} T^{\prime}\right\rangle$ rather than mean convection.

The budget for the turbulent heat flux $\left\langle u_{i}^{\prime} T^{\prime}\right\rangle$ is given as,

$\mathscr{C}_{\left\langle u_{i}^{\prime} T^{\prime}\right\rangle}=\mathscr{P}_{\left\langle u_{i}^{\prime} T^{\prime}\right\rangle}+\varepsilon_{\left\langle u_{i}^{\prime} T^{\prime}\right\rangle}+\mathscr{T}_{\left\langle u_{i}^{\prime} T^{\prime}\right\rangle}+\Psi_{\left\langle u_{i}^{\prime} T^{\prime}\right\rangle}+\mathscr{D}_{\left\langle u_{i}^{\prime} T^{\prime}\right\rangle}$

where

$$
\begin{aligned}
& \mathscr{C}_{\left\langle u_{i}^{\prime} T^{\prime}\right\rangle}=\left\langle u_{j}\right\rangle \frac{\partial\left\langle u_{i}^{\prime} T^{\prime}\right\rangle}{\partial x_{j}} \\
& \mathscr{P}_{\left\langle u_{i}^{\prime} T^{\prime}\right\rangle}=-\left[\left\langle u_{j}^{\prime} T^{\prime}\right\rangle \frac{\partial\left\langle u_{i}\right\rangle}{\partial x_{j}}+\left\langle u_{i}^{\prime} u_{j}^{\prime}\right\rangle \frac{\partial\langle T\rangle}{\partial x_{j}}\right] \\
& \varepsilon_{\left\langle u_{i}^{\prime} T^{\prime}\right\rangle}=-\left(\frac{1}{R e}+\frac{1}{R e P r}\right)\left\langle\frac{\partial T^{\prime}}{\partial x_{j}} \frac{\partial u_{i}^{\prime}}{\partial x_{j}}\right\rangle \\
& \mathscr{T}_{\left\langle u_{i}^{\prime} T^{\prime}\right\rangle}=-\frac{\partial\left\langle u_{i}^{\prime} u_{j}^{\prime} T^{\prime}\right\rangle}{\partial x_{j}} \\
& \Psi_{\left\langle u_{i}^{\prime} T^{\prime}\right\rangle}=-\left\langle T^{\prime} \frac{\partial p^{\prime}}{\partial x_{i}}\right\rangle \\
& \mathscr{D}_{\left\langle u_{i}^{\prime} T^{\prime}\right\rangle}=\frac{1}{R e}\left[\frac{\partial}{\partial x_{j}}\left\langle T^{\prime} \frac{\partial u_{i}^{\prime}}{\partial x_{j}}\right\rangle\right] \\
& +\frac{1}{\operatorname{RePr}}\left[\frac{\partial}{\partial x_{j}}\left\langle u_{i}^{\prime} \frac{\partial T^{\prime}}{\partial x_{j}}\right\rangle\right]
\end{aligned}
$$


Fig. 20(a) and (b) shows the inner scaled budgets for the turbulent heat fluxes $\left\langle u^{\prime} T^{\prime}\right\rangle$ and $\left\langle v^{\prime} T^{\prime}\right\rangle$, respectively. The production for $\left\langle u^{\prime} T^{\prime}\right\rangle$ is negative. The molecular diffusion term is significant in the near wall region, where it balances the high dissipation value. The production term is balanced by all the other terms of the budget. The temperature-pressure diffusion term becomes larger than the dissipation beyond $y^{+}=20.0$. The wall normal heat flux $\left\langle v^{\prime} T^{\prime}\right\rangle$ budget shows that the dissipation and molecular diffusion are negligible except in the viscous sublayer. The convection term is also insignificant. The production is balanced by the temperature-pressure diffusion term. It can be observed generally, that the temperature-pressure diffusion term is always on the loss side, balancing production for both turbulent heat fluxes. This behaviour is similar to turbulent boundary layers (Li et al., 2009).

\section{Conclusions}

Direct numerical simulation of a wall jet at $R e=7500$, with heat transfer from an iso-thermal wall, is performed. The resulting mean flow and Reynolds stresses compare well with the available data from various wall jet studies. The jet spreading rate, maximum velocity decay and wall shear stress are also compared with the available data.

Mean heat transfer properties in terms of Stanton number St, Nusselt number $\mathrm{Nu}$ and velocity-temperature correlation $\rho_{u T}$ are presented and compared with the existing data. The Nusselt number follows the empirical correlation $N u R e^{-0.8}=C(x / h)^{-0.6}$, with $C=0.07-0.115 . \rho_{u T}<1.0$ near the wall, which indicates that the fully developed boundary layer state has not been achieved at the given Reynolds number and that the outer layer is influencing the inner layer.

The turbulent Prandtl number $P r_{t}$ is not constant in the near wall region $y^{+}<100$. It fluctuates between large negative and positive values around the maximum velocity location.

The scaling properties of velocity and heat transfer parameters are presented. The mean temperature $\langle T\rangle, T_{r m s}$, streamwise $\left\langle u^{\prime} T^{\prime}\right\rangle$ and wall normal $\left\langle v^{\prime} T^{\prime}\right\rangle$ heat flux profiles collapse with inner, outer and thermal scaling.

The temperature variance $\left\langle T^{\prime} T^{\prime}\right\rangle$ and heat flux $\left\langle u_{i}^{\prime} T^{\prime}\right\rangle$ budgets are presented. For the $\left\langle v^{\prime} T^{\prime}\right\rangle$ budget production is balanced by the pressure-temperature diffusion term, which is identical to turbulent boundary layer behaviour.

\section{References}

AbdulNour, R.S., Willenborg, K., McGrath, J.J., Foss, J.F., AbdulNour, B.S., 2000. Measurements of the convection heat transfer coefficient for a planar wall jet: uniform temperature and uniform heat flux boundary conditions. Exp. Thermal Fluid Sci. 22 (3-4), 121-131.
Abrahamsson, H., Johansson, B., Lofdahl, L., 1994. A turbulent plane two-dimensional wall jet in a quiescent surrounding. Eur. J. Mech. B. Fluids 13, 533-556.

Ahlman, D., Brethouwer, G., Johansson, A., 2007. Direct numerical simulation of a plane turbulent wall-jet including scalar mixing. Phys. Fluids 19, 065102.

Akfirat, J.C., 1966. Transfer of heat from an isothermal flat plate to a two-dimensional wall jet. In: Proceedings of the Third International Heat Transfer Conference, 2, pp. 274-279.

Antonia, R.A., Krishnamoorthy, L.V., Fulachier, L., 1988. Correlation between the longitudinal velocity fluctuation and temperature fluctuation in the near-wall region of a turbulent boundary layer. Int. J. Heat Mass Transf. 31 (4), 723-730.

Banyassady, R., Piomelli, U., 2014. Turbulent plane wall jets over smooth and rough surfaces. J. Turbul. 15 (3), 186-207.

Banyassady, R., Piomelli, U., 2015. Interaction of inner and outer layers in plane and radial wall jets. J. Turbul. 16 (5), 460-483.

Bremhorst, K., Bullock, K.J., 1970. Spectral measurements of temperature and longitudinal velocity fluctuations in fully developed pipe flow. Int. J. Heat Mass Transf. 13, 1313-1329.

Dacos, T., Verriopoulos, C., Gibson, M., 1984. Turbulent flow with heat transfer in plane and curved wall jet. J. Fluid Mech. 145, 339-360.

Dejoan, A., Leschziner, M.A., 2005. Large eddy simulation of a plane turbulent wall jet. Phys. Fluids 17, 025102

Eriksson, J., Karlsson, R., Persson, J., 1998. An experimental study of a two-dimensional plane turbulent wall jet. Exp. Fluids 25, 50-60.

George, W., Abrahamsson, H., Eriksson, J., Karlsson, R., Lofdhal, L., Wosnik, M., 2000. A similarity theory for the turbulent plane wall jet without external stream. J. Fluid Mech. 425, 367-411.

Hunt, J.C.R., Wray, A.A., Moin, P., 1988. Eddies, streams, and convergence zones in turbulent flows. In: Proceedings of the 1988 Summer Program Studying Turbulence Using Numerical Simulation Databases, Vol. 2. Stanford University, pp. 193-208.

Iritani, Y., Kasagi, N., Hirata, M., 1985. Heat transfer mechanism and associated turbulence structure in the near-wall region of a turbulent boundary layer. In: Bradbury, L.J.S., Durst, F., Launder, B.E., Schmidt, F.W., Whitelaw, J.H. (Eds.), Turbulent Shear Flows 4. Springer-Verlag, Berlin, pp. 223-234.

Kader, B.A., Yaglom, M., 1972. Heat and mass transfer laws for fully turbulent wall flows. Int. J. Heat Mass Transf. 15, 2329-2351.

Kline, S.J., Reynolds, W.C., Schraub, F.A., Runstadler, P.W., 1967. The structure of turbulent boundary layers. J. Fluid Mech. 30, 741-773.

Kong, H., Choi, H., Lee, J.S., 2000. Direct numerical simulation of turbulent thermal boundary layers. Phys. Fluids 12, 2555.

Launder, B., Rodi, W., 1983. The turbulent wall jet measurements and modeling. Annu. Rev. Fluid Mech. 15, 429-459.

Leonard, B.P., 1979. A stable and accurate convective modelling procedure based on quadratic upstream interpolation. Comput. Methods Appl. Mech. Eng. 19, 59-98.

Li, Q., Schlatter, P., Brandt, L., Henningson, D., 2009. DNS of a spatially developing turbulent boundary layer with passive scalar transport. Int. J. Heat Fluid Flow 30, 916-929.

Mabuchi, I., Kumada, M., 1972. Studies on heat transfer to turbulent jets with adjacent boundaries. Bull. JSME 15 (88), 1236-1245.

Naqavi, I.Z., Tucker, P.G., Liu, Y., 2014. Large-eddy simulation of the interaction of wall jets with external stream. Int. J. Heat Fluid Flow 50, 431-444.

Nizou, P.Y., 1981. Heat and momentum transfer in a plane turbulent wall jet. Trans. ASME C: J. Heat Transf. 103, 138-140.

Pouransari, Z., Biferale, L., Johansson, A.V., 2015. Statistical analysis of the velocity and scalar fields in reacting turbulent wall- jets. Phys. Fluids 27, 025102.

Radhakrishnan, S., Keating, A., Piomelli, U., Silva Lopes, A., 2006. Large-Eddy Simulations of High Reynolds-Number Flow Over a Contoured Ramp. AIAA Paper 2006-899.

Rostamy, N., Bergstrom, D., Sumner, D., Bugg, J., 2011. The effect of surface roughness on the turbulence structure of a plane wall jet. Phys. Fluids 23, 085103.

Wardana, I.N.G., Ueda, T., Mizomoto, M., 1995. Velocity-temperature correlation in strongly heated channel flow. Exp. Fluids 18, 454-461. 